these measurements the following conclusions can be drawn:

1. The value of the surface anisotropy obtained from our measurements is in good agreement with the theoretically computed value 10) and with the exchange surface anisotropy 11) and has the order of $K_{\mathrm{S}}$ observed experimentally by means of thin films spin-wave resonance 12 ).

2. The irregularities of the surface play a minor role in line broadening as shown by the small difference between whiskers and electrolytically polished crystals having largely differing surface states.

3. The line broadening due to an increase of the measured area indicates that here the inhomogeneity of the demagnetising field begins to assert itself.

4. The agreement between the theory referred to above and the experimental results on bulk silicon iron single crystals is good. It is proof that, at least in some metallic ferromagnets having few imperfections, the intrinsic resonance linewidth (without skin effect and surface anisotropy broadening) is under 10 Oe.

\section{References}

1) D.S. Rodbell, J. Appl. Phys. 30 (1959) $187 \mathrm{~S}$.

2) Z. Frait, Czech. J. Phys. 10 (1960) 546.

3) W.S.Ament and G.T.Rado, Phys. Rev. 97 (1955) 1558.

4) J.R. Macdonald, Phys. Rev. 103 (1956) 280. M.I. Kaganov and J.Lu, Izv. Akad. Nauk SSSR, ser. fiz. 25 (1961) 1375.

5) Z. Frait, Czech. J. Phys., to be published, preprints available.

6) A.F.Kip and R.D.Arnold, Phys. Rev. 75 (1949) 1556. A.Strub, J. phys, radium 21 (1960) $43 \mathrm{~A}$.

7) Z. Frait and B.Heinrich, Arch. Sci. 14 (1962) $138 \mathrm{~S}$.

8) Z. Frait, Czech. J. Phys. 9 (1959) 403; Phys. stat. sol. 2 (1962) 1417.

9) Z.Frait and M. Ondris, Phys. stat. sol. 2 (1962) K185.

10) L. Neel, J. phys, radium 15 (1954) 225.

11) W.H. Meiklejohn, J. Appl. Phys. 33 (1962) 1328S.

12) C. F. Kooi et al., J. Phys. Soc. Japan 17, suppl. BI (1962) 599 .

\title{
A RESTRICTED CLASS OF EXACT EIGENSTATES OF THE PAIRING-FORCE HAMILTONIAN *
}

\author{
R. W. RICHARDSON \\ H.M. Randall Laboratory of Physics, \\ University of Michigan, Ann Arbor, Michigan
}

Received 23 November 1962

In this note we will show that there exists a restricted class of eigenstates of the pair-force Hamiltonian which contain $N$ pairs of particles and which are natural generalisations of those states which contain one pair. The wave function of these $N$-pair states has the form of an antisymmetrised product of $N$ 1-pair wave functions and the energy is a sum of $N 1$-pair energies. These 1 -pair wave functions and energies are obtained from a 1-pair Schrodinger equation in which the pairing interaction has been replaced by an effective pairing interaction which in turn depends upon the $N 1$-pair energies. These eigenstates may be used to study the effects of including a pairing interaction in the Nilsson model of the atomic nucleus 1) and also in some nuclei near closed shells. They may also be used to evaluate the accuracy of the methods of the theory of superconductivity which have been used by many authors 2,3 ) to include pairing forces in an independent particle model of the nucleus.

We split the pairing-force Hamiltonian into that part which describes unpaired (i.e., noninteracting) particles and that part which describes the paired particles. Thus

$$
H=H_{1}+H_{2} \text {, }
$$

where

$$
H_{1}=\sum_{\left(f \in S_{1}\right)} 2 \epsilon_{f} N_{f} \text {, }
$$

$$
H_{2}=\sum_{\left(f \in S_{2}\right)} 2 \epsilon_{f} N_{f}-g \sum_{\left(f \in S_{2}\right)} \sum_{\left(f^{\prime} \in S_{2}\right)} b_{f}^{+} b_{f^{\prime}},
$$

and where $\sigma_{f}$ is the energy of the single-particle state $(f, \sigma)$ (where $\sigma= \pm$ denotes states which are conjugate with respect to time reversal), $S_{1}$ is the set of states outside a finite energy interval about the Fermi energy (which is specified by the interaction) plus those states in this interval that are occupied by unpaired particles, $S_{2}$ is the finite set of states not included in $S_{1}$,

$$
\begin{gathered}
N_{f}=\frac{1}{2}\left(a_{f+}^{+} a_{f+}+a_{f-}^{+} a_{f-}\right), \\
b_{f}=a_{f-} a_{f+},
\end{gathered}
$$

* Supported by the National Science Foundation. 
and the $a^{+1} s$ and $a^{\text {'s }}$ are fermion creation and annihilation operators.

Since $H_{1}$ and $H_{2}$ refer to different dynamical variables of the system, the eigenfunctions of $H$ are products of an eigenfunction of $\mathrm{H}_{1}$ with one of $\mathrm{H}_{2}$ and the energy is a sum of the corresponding energy eigenvalues. Since $H_{1}$ is already diagonal, we will only consider $\mathrm{H}_{2}$ and its eigenfunctions and we will restrict $f$ to tho se values contained in $S_{2}$.

The operators $N_{f}$ and $b_{f}$ satisfy the commutation relations

$$
\begin{aligned}
& {\left[b_{f}, N_{f^{\prime}}\right]=\delta_{f f^{\prime}} b_{f}} \\
& {\left[b_{f}, b_{f^{+}}^{+}\right]=\sigma_{f f}\left(1-2 N_{f}\right) .}
\end{aligned}
$$

If we could neglect the term $2 N_{f}$ on the right hand side of (7), we would have boson commutation relations and the diagonalisation of $\mathrm{H}_{2}$ would be straightforward. The presence of the term, $2 N_{f}$, reflects the fact thet the $b^{\prime}$ s are products of Fermi operators which must obey the Pauli principle.

We now formulate a method in which the complicating term, $2 N_{f}$, in (7) gives no contribution to the calculation of the matrix elements of $\mathrm{H}_{2}$.

i. We first expand an eigenstate of $\mathrm{H}_{2}$ as

$$
|\psi\rangle=(N !)^{-\frac{1}{2}} \sum_{f_{1} \ldots f_{N}} \psi\left(f_{1} \ldots f_{N}\right) b_{f_{1}}^{+} \ldots b_{f_{N}}^{+}|0\rangle \text {, }
$$

where $|0\rangle$ is the vacuum state.

ii. We can impose two requirements on $\psi\left(f_{1} \ldots f_{N}\right)$

1. Since $\left[b_{f}^{+}, b_{f}^{+}\right]=0$, we require $\psi\left(f_{1} \ldots f_{N}\right)$ to be a symmetric function of its arguments.

2. Since $b_{f}^{2}=0$, the values of $\psi\left(f_{1} \ldots f_{N}\right)$ with two or more arguments equal are without physical significance. We are therefore free to choose these values of $\psi$ in any convenient way. A convenient choice is zero.

These two conditions may be satisfied by letting

$$
\psi\left(f_{1} \ldots f_{N}\right)=\theta\left(f_{1} \ldots f_{N}\right) \varphi\left(f_{1} \ldots f_{N}\right),
$$

where $\varphi$ is a symmetric function of its arguments and

$$
\begin{aligned}
\theta\left(f_{1} \ldots f_{N}\right) & =1, & \text { if } \quad f_{i} \neq f_{j}, & \text { all } i \neq j, \\
& =0, & \text { if any } f_{i}=f_{j}, & i \neq j .
\end{aligned}
$$

In general it may be verified that

$$
\theta\left(f_{1} \ldots f_{N}\right)=\prod_{i<j}\left(1-\delta_{f_{i} f_{j}}\right) .
$$

iii. For $\psi^{\prime} s$ of the form (9) the normalisation is

$$
\langle\psi \mid \psi\rangle=\sum_{f_{1} \ldots f_{N}}\left|\psi\left(f_{1} \ldots f_{N}\right)\right|^{2}=1
$$

and the expectation value of $\mathrm{H}_{2}$ is

$$
\begin{array}{r}
\left\langle\psi\left|H_{2}\right| \psi\right\rangle=\sum_{f_{1} \ldots f_{N}}\left(2 \epsilon_{f_{1}}+\ldots+2 \epsilon_{f_{N}}\right)\left|\psi\left(f_{1} \ldots f_{N}\right)\right|^{2} \\
-g \sum_{i=1}^{N} f \ldots f_{i-1} \sum_{f_{i+1}} \ldots f_{N} f f^{\prime} \psi^{*}\left(f_{1} \ldots f_{i-1} f f_{i+1} \ldots f_{N}\right) \\
\quad \times \psi\left(f_{1} \ldots f_{i-1} f^{\prime} f_{i+1} \ldots f_{N}\right) .(12)
\end{array}
$$

Note that these are the same results that would have been gotten using boson commutation relations for the $b^{\prime}$ s by ignoring the term $2 N_{f}$ in (7).

iv. We now determine the exact eigenstates of $\mathrm{H}_{2}$ by requiring $\psi$ to satisfy the normalisation condition (11) and the Schroddinger equation derived from the variational equations

$$
\frac{\delta\left\langle\psi\left|H_{2}-E\right| \psi\right\rangle}{\delta \psi^{*}\left(f_{1} \ldots f_{N}\right)}=\frac{\delta\left\langle\psi\left|H_{2}-E\right| \psi\right\rangle}{\delta \varphi\left(f_{1} \ldots f_{N}\right)}=0 .
$$

Using (9), (12) and $\theta^{2}=\theta$, (13) becomes

$$
\begin{gathered}
\left(2 \epsilon_{1}+\ldots+2 \epsilon_{f_{N}}-E\right) \theta\left(f_{1} \ldots f_{N}\right) \varphi\left(f_{1} \ldots f_{N}\right) \\
-g \sum_{i=1}^{N} \sum_{f} \theta\left(f_{1} \ldots f_{N}\right) \theta\left(f_{1} \ldots f_{i-1} f f_{i+1} \ldots f_{N}\right) \\
\quad \times \varphi\left(f_{1} \ldots f_{i-1} f f_{i+1} \ldots f_{N}\right)=0 .
\end{gathered}
$$

Since we obtain the same equation for $\varphi^{*}$, we can choose $\varphi$ to be real. From the definition of $\theta$ it follows that

$$
\begin{aligned}
\theta\left(f_{1} \ldots f_{N}\right) \theta\left(f_{1} \ldots f_{i-1} f f_{i+1} \ldots f_{N}\right) & \\
& =\theta\left(f_{1} \ldots f_{N}\right)\left[1-\sum_{\substack{j=1 \\
j \neq i}}^{N} \delta_{f_{j}}\right] .
\end{aligned}
$$

Therefore $\varphi$ satisfies (14) if

$$
\begin{aligned}
& \left(2 \epsilon_{f_{1}}+\ldots+2 \epsilon_{f_{N}}-E\right) \varphi\left(f_{1} \ldots f_{N}\right) \\
& -g \sum_{i=1}^{N} \sum_{f}\left[1-\sum_{\substack{j=1 \\
(j \neq i)}}^{N} \delta_{j} f \varphi\left(f_{1} \ldots f_{i-1} f f_{i+1} \ldots f_{N}\right)=0 .\right.
\end{aligned}
$$

For $N=1,(16)$ may be solved without any restrictions. For, in this case (16) becomes

$$
\left(2 \epsilon_{f_{1}}-E\right) \varphi\left(f_{1}\right)-g \Sigma_{f} \varphi(f)=0 .
$$

The solutions of (17) are

$$
\varphi_{p_{1}}\left(f_{1}\right)=g C_{p_{1}}\left(2 \epsilon_{f_{1}}-E_{p_{1}}\right)^{-1}
$$

where $g C_{p_{1}}$ is a normalisation constant and $E=E_{p_{1}}$, where $E_{p 1}$ is a root of the equation

$$
1=g \Sigma_{f}\left(2 \epsilon_{f}-E_{p_{1}}\right)^{-1}
$$

For $N>1$, we will show that there exists a restricted class of solutions of (16) which are natural generalisations of (18) and (19), i.e., 


$$
\begin{aligned}
& \varphi_{p_{1}} \ldots p_{N}\left(f_{1} \ldots f_{N}\right) \\
& \quad=g^{N} C_{p_{1}} \ldots p_{N} \sum_{P} P\left(\prod_{k=1}^{N}\left(2 \epsilon_{f_{k}}-E_{p_{k}}\right)^{-1}\right),
\end{aligned}
$$

where $g^{N} C_{p_{1}} \ldots p_{N}$ is a normalisation constant, $\Sigma_{P} P$ is a sum over the $N$ ! permutations of the indices $\left(p_{1} \ldots p_{N}\right)$, and

$$
E=\sum_{i=1}^{N} E_{p_{i}} .
$$

The $E_{p_{i}}(i=1 \ldots N)$ are $N$ distinct roots of the coupled equations

$$
.1=g_{i} \Sigma_{f}\left(2 \epsilon_{f}-E_{p_{i}}\right)^{-1}, \quad i=1 . . . N,
$$

where

$$
g_{i}=g\left(1+2 g \sum_{\substack{j=1 \\(j \neq i)}}^{N} \frac{1}{E_{p_{j}}-E_{p_{i}}}\right)^{-1} .
$$

This may be verified by direct substitution of (20) into (16). However, the restriction

$$
E_{p_{i}} \neq E_{p_{j}}, \quad \text { all } i \neq j,
$$

is essential to the derivation of (22) and (23).

Given a single-particle spectrum, $\epsilon$, and an interaction strength, $g$, the solution of eq. (22) proceeds in two steps:

1. One must first decide whether the restrictions (24) on the $E_{p_{i}}$ are compatible with the $E_{p_{i}}$ satisfying (22). This must be carefully checked for each particular problem. However, for the Nilsson model of the atomic nucleus 1) (whose single-particle spectrum satisfies $\epsilon_{f} \neq \epsilon_{f}$ for $f \neq f^{\prime}$ ), we may choose the indices $p_{i}$ so that

$$
\lim _{g \rightarrow 0^{+}} E_{p_{i}}=2 \epsilon_{p_{i}},
$$

and therefore (24) is satisfied in this limit. Study of eqs. (22) then indicates that there is a range of $g, g>0$, for which (22) and (24) are compatible. These same considerations hold true for any state of a system for which

$$
\lim _{g \rightarrow 0^{+}} E_{p_{i}} \neq \lim _{g \rightarrow 0^{+}} E_{p_{j}}, \quad i \neq j
$$

Thus, there exists a large class of systems and interaction strengths for which (22) and (24) are compatible.

2. For systems for which (22) and (24) are compatible, eqs. (22) may be solved numerically for the $E_{p_{i}}$ The solution of eqs. (22) is greatly facilitated by the presence of the same function,

$$
F(E)=\Sigma_{f}\left(2 \epsilon_{f}-E\right)^{-1},
$$

evaluated for different values of its argument in each equation. This function may be calculated once and then eqs. (22) may be solved by iteration.
Some of the advantages of this method over existing work (for example, see ref. 4)) on the exact diagonalisation of the pairing-force Hamiltonian are:

1. The computational effort needed to solve (22) is in general much less than that needed to perform a direct diagonalisation of $\mathrm{H}_{2}$.

2. General properties of the eigenvalues of $\mathrm{H}_{2}$ may be studied by considerings eqs. (21) and (22). For example, the pairing energy can be related directly to $g$. The pairing energy is defined as

$$
P(2 N)=2 E(2 N-1)-E(2 N)-E(2 N-2),
$$

where $E(2 N-\nu)$ is the ground state energy of $2 N-\nu$ particles. Note that this definition of $P(2 N)$ assumes that $E(2 N-\nu)$ is a linear function of $\nu$ plus a discontinuous pairing term and that it neglects quadratic terms in. $\nu$. If we assume that the energies of the paired particles in the three ground states in (25) are given by (21), then we obtain the total energies of the states by adding the energy of the unpaired particles to that of the paired particles, i.e.,

$$
\begin{aligned}
& E(2 N-2)=\sum_{i=1}^{N-1} E_{p_{i}(2 N-2)}, \\
& E(2 N-1)=\sum_{i=1}^{N-1} E_{p_{i}(2 N-1)+\epsilon}, \\
& E(2 N)=\sum_{i=1}^{N} E_{p_{i}(2 N)},
\end{aligned}
$$

where $\epsilon_{F}$ is the energy of the last filled level in the ground state of $2 N$ noninteracting particles, $E_{p_{i}}(2 N-\nu)$ is the energy of the $i$-th pair in the ground state of $2 N-\nu$ interacting particles, and $p_{N}=F$. Substituting the se expressions into (25) and again neglecting terms quadratic in $\nu$, we have

$$
P(2 N)=2 \epsilon_{F}-E_{F}(2 N) .
$$

Thus, if $P(2 N)$ is given, then $E_{F}$ is known and the $N$ unknowns in eqs. (22) are $g$ and $E_{p_{i}}$,
$i=1$...N-1.

3. The wave function is given as a rational function of the $N$ pair energies $E_{p_{i}}$ which may be calculated as accurately as is desired.

We are now performing detailed applications of these equations and the results will be reported at a later date.

1) S.G. Nilsson, Dan. Mat. Fys. Medd. 29, no. 16 (1955).

2) L.S. Kisslinger and R.A.Sorensen, Dan. Mat. Fys. Medd. 32 no. 9 (1960).

3) V.G.Solov'ev, Thesis, Dubna USSR (1961).

4) A.K.Kerman et al., Phys. Rev. 124 (1961) 162. 